\title{
Evaluacion por Western Blot, Inmunofluorescencia Indirecta y ELISA de Perros Infectados con Leishmania (Leishmania) infantum
}

\author{
Western blot, ELISA and indirect immunofluorescence test \\ evaluation of Leishmania (Leishmania) infantum-infected dogs \\ Jimmy J. Vargas-Duarte1, Myriam C. López-Páez², Jesús E. Escovar-Castro ${ }^{3}$ \\ y José Fernández-Manrique ${ }^{3}$
}

1 Instituto de Genética, Universidad Nacional de Colombia. jjvargasd@unal.edu.co.

2 Departamento de Salud Pública, Parasitología. Facultad de Medicina, Universidad Nacional de Colombia..mclopezp@unal.edu.co.

3 Departamento de Ciencias básicas. Universidad de la Salle. jeescobar@unisalle.edu.co, josefernandezm@yahoo.com

Recibido 19 Enero 2009/Enviado para Modificación 9 Julio 2009/Aceptado 15 Julio 2009

\section{RESUMEN}

Objetivo Evaluar el desempeño de las pruebas empleadas en Colombia para el diagnóstico de la leishmaniasis visceral canina y adaptar una técnica de Western blot empleando animales experimental y naturalmente infectados.

Metodología Se obtuvieron sueros de 10 perros infectados experimentalmente con $L$. infantum, 5 perros infectados naturalmente, 16 perros sanos, 26 de reacción cruzada (infectados con Babesia canis, Erhlichia canis, Dirofilaria immitis, Trypanosoma cruzi, Leishmania (Viannia) spp.), 40 de zonas no endémicas y 150 de zona endémica. Todos fueron evaluados mediante las pruebas de inmunofluorescencia indirecta (IFI), ELISA y Western blot (WB).

Resultados Se encontró que IFI tuvo el mayor porcentaje de positividad en los perros infectados (73\%) mientras que el menor porcentaje de falsos positivos se obtuvo por WB $(2,5 \%)$. La prueba de ELISA fue la menos eficiente. Fueron reconocidas 24 fracciones antigénicas, las bandas de 29, 34, 50, 69, 75, 86, 99 y 123 kDa fueron responsables de reacciones inespecíficas en los sueros de perros sanos, de zona no endémica y de reacción cruzada. Las bandas por debajo de 29 kDa mostraron ser potencialmente diagnósticas, especialmente la fracción de $13 \mathrm{kDa}$.

Conclusiones Los métodos directos y serológicos pueden subdiagnosticar la infección por Leishmania, solamente un constructo que combine tanto pruebas directas como indirectas sería la forma más eficiente de diagnóstico.

Palabras Clave: Leishmaniasis Visceral, Western blotting, prueba ELISA, pruebas inmunológicas, reservorios (fuente: DeCS, BIREME). 


\section{ABSTRACT}

Objective Evaluating canine visceral leishmaniasis diagnostic test performance in Colombia and adapting the Western blot test in naturally and experimentally infected dogs.

Methods Sera were obtained from 10 experimentally L. Infantum-infected dogs, 5 naturally infected dogs, 16 healthy dogs, 26 Babesia canis, Erhlichia canis, Dirofilaria immitis, Trypanosoma cruzi and Leishmania (Viannia) spp infected dogs, 40 dogs from non-endemic areas and 150 from endemic areas. Sera were tested for $L$. infantum infection using immunofluorescent antibody (IFAT), ELISA and Western blot (WB) tests. Results Positives results were obtained for $73 \%$ of known infected dogs by the IFAT test and false positives were obtained for $2.5 \%$ of non-infected dogs using WB. ELISA was not efficient for diagnosis. 24 antigenic fractions were recognised in tested sera using WB; however, 29, 34, 50, 69, 75, 86, 99 and $123 \mathrm{kDa}$ bands were recognised in sera from dogs from non-endemic areas, healthy dogs and Trypanosoma cruzi, Erhlichia canis, Dirofilaria immitis and Babesia canis infected dogs. The $13 \mathrm{kDa}$ fraction proved potentially useful for diagnosing canine visceral leishmaniasis.

Conclusions The separate use of parasitological and serological test could lead to misdiagnosis of Leishmania infection; using both kinds of technique simultaneously is thus highly recommended.

Key Words: Leishmaniasis, immunoblotting, enzyme-linked immunosorbent assay (ELISA), fluorescent antibody technique, immunological test, reservoir (source: $\mathrm{MeSH}$, NLM).

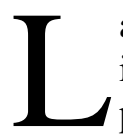

a leishmaniasis visceral (LV) es una de las enfermedades parasitarias más importantes en humanos y su transmisión en América se relaciona con la presencia de reservorios animales (1). Durante los últimos 30 años se han incrementado los casos de LV debido a factores como malnutrición, el síndrome de inmunodeficiencia adquirida (SIDA), cambios demográficos y ecológicos, incremento de cepas resistentes a los tratamientos, establecimiento de nuevos mecanismos de transmisión y fallas en las estrategias convencionales de control de reservorios y flebótomos $(2,3)$.

En los perros la LV induce un cuadro clínico variable con manifestaciones tanto cutáneas como viscerales, sin embargo, del 35 al $59 \%$ de los animales infectados son asintomáticos lo que plantea un importante problema de salud pública (4). La infección en el perro, principal reservorio doméstico de LV, facilita la transmisión vectorial al hombre, mediante mosquitos flebótomos contaminados con los amastigotes presentes en la sangre y la piel de este tipo de mascotas $(1,5)$. Las pruebas serológicas han sido las herramientas de elección en el diagnóstico de LV canina, especialmente cuando se requiere detectar portadores asintomáticos o monitorear esquemas de control (6-9). Se han reportado valores entre 90 - $100 \%$ de sensibilidad y $80 \%$ de especificidad para 
inmunofluorescencia indirecta (IFI) (3,9-10), del $85-96 \%$ de sensibilidad y 86 $98 \%$ de especificidad para las pruebas de ELISA $(4,6,10-13)$ y con respecto a la prueba de aglutinación directa (DAT) se habla de una sensibilidad del $100 \%$ y una especificidad del 98,8 \% (14). Pruebas serológicas como el Western blotting (WB), son consideradas herramientas altamente sensibles y que han mejorado el diagnóstico de LV en perros, sin embargo, no existe aún consenso con respecto al patrón de reconocimiento de polipéptidos que pueda relacionarse con la dinámica clínica o parasitológica durante la infección en el perro (3,7,8,15-24).

El presente trabajo evaluó el desempeño de las diferentes pruebas empleadas en el diagnóstico de LV canina, con el propósito de reforzar las estrategias de control en reservorios y mediante la adaptación de la prueba de WB buscó establecer el grado de correlación presente entre los resultados obtenidos y los estados clínico y parasitológico.

\section{MATERIALES Y METODOS}

Perros inoculados experimentalmente con Leishmania infantum: Se inocularon 2 grupos de 3 perros cada uno con $10^{6}$ y $10^{4}$ promastigotes de la cepa MCAN/ COL/98/CAT por vía intravenosa (IV) respectivamente y un grupo de 4 perros con $10^{4}$ parásitos vía intradérmica (ID). Todos fueron sometidos a los 2,4 y 8 meses post inoculación (mpi) a valoración clínica, toma de sangre, aspirados de ganglio linfático poplíteo y cultivo en medio $3 \mathrm{~N}$. Los sueros fueron alicuotados y almacenados a $-20^{\circ} \mathrm{C}$.

Perros inoculados experimentalmente con Trypanosoma cruzi: Se inocularon 3 perros con tripomastigotes de la cepa MHOM/CO/SPR y 2 perros con la cepa $\mathrm{MHOM} / \mathrm{CO} / \mathrm{DA}$ por vía IV con $2 \times 10^{4}$ parásitos por kg de peso. Todos los perros fueron sometidos a los 15 días, 1,2 y 4 meses post inoculación a valoración clínica, toma de sangre de la vena cefálica para prueba de microhematocrito y para la obtención de sueros.

Sueros controles positivos y negativos: Se tomaron 5 sueros de perros de zona endémica y 16 sueros de perros de la ciudad de Bogotá D.C. Todos fueron confirmados parasitológicamente por impronta y cultivo de muestras de ganglio linfático poplíteo, hígado, bazo y médula ósea.

Sueros de reacción cruzada con otros parásitos: Se recolectaron 10 muestras de sueros de perros infectados con Babesia spp. (3), Erhlichia spp. (3), Dirofilaria spp. (2) y Leishmania (Viannia) spp. (2). 
Sueros de perros de zona endémica y no endémica: Se colectaron 150 y 40 muestras de sangre en zona endémica y no endémica respectivamente.

Prueba de Inmunofluorescencia indirecta: Se llevó a cabo teniendo en cuenta lo descrito por Corredor et al. (25) empleando promastigotes de Leishmania infantum y un conjugado anti-Ig $\mathrm{G}$ canina marcado con fluoresceína a la dilución de 1:32. Se emplearon diluciones seriadas de los sueros desde 1:16 hasta 1:4096 y el punto de corte fue establecido en una dilución $\geq 1: 32$.

Prueba de ELISA: Se llevó a cabo teniendo en cuenta lo descrito por Pappas et al. (26) y Vega et al. (10) empleando un lisado de promastigotes de Leishmania infantum con una concentración de 7,5 $\mu / \mathrm{ml}$ y diluciones de suero de 1:600. Se empleó un conjugado anti-Ig G canina marcado con fosfatasa alcalina a la dilución 1:6000. El punto de corte fue establecido a un valor de absorbancia $\geq$ 0.421 .

Prueba de Western Blotting (WB): Se llevó a cabo siguiendo lo descrito por Laemmli (27) y Towbin et al. (28) empleando un lisado de promastigotes de Leishmania infantum cuya concentración de proteína fue medida por el método de Bradford (29) empleando 5 ìg por carril. La separación por electroforesis se llevó a cabo en geles de poliacrilamia al $12 \%$ con SDS 0,1\%. La dilución de suero empleada fue de 1:200 y del conjugado de 1:20000.

Análisis de resultados: Se calcularon los parámetros de sensibilidad, especificidad, valor predictivo positivo, valor predictivo negativo y prevalencia aparente para cada una de las pruebas así como la concordancia entre ellas con la sintomatología clínica tanto a nivel experimental como a nivel de campo empleando el programa WinEpiscope 2.0 (Borland® \& Delphi TM, 1998).

Aspectos éticos: Se aplicó la Ley 84 de 1989 (30) y la Resolución No. 8430 de 1993 del Ministerio de Salud (31).

\section{RESULTADOS}

Los perros inoculados experimentalmente mostraron variaciones en la respuesta clínica y parasitológica dependiendo del tipo de inóculo y de la fase de infección. En los inóculos $10^{6}$ y $10^{4} \mathrm{IV}$ se observaron perros sintomáticos y asintomáticos mientras que con el inóculo $10^{4} \mathrm{ID}$ todos los perros en algún momento fueron sintomáticos. No se logró demostrar la presencia de amastigotes en 3 de los 10 perros inoculados experimentalmente (Tabla 1). 
Tabla 1. Resultados obtenidos en los perros infectados experimental y naturalmente a nivel clínico, parasitológico y serológico

\begin{tabular}{|c|c|c|c|c|c|c|}
\hline $\begin{array}{c}\text { Inóculo } \\
\text { Experimental }\end{array}$ & $\begin{array}{c}\text { Perro } \\
\text { No. }\end{array}$ & $\begin{array}{c}\text { Fase } \\
\text { infección } \\
\text { experimental }\end{array}$ & $\begin{array}{c}\text { Presencia } \\
\text { de signos } \\
\text { clínicos }\end{array}$ & $\begin{array}{c}\text { Examen } \\
\text { parasitológico }\end{array}$ & $\begin{array}{c}\text { Titulo } \\
\text { IFI }\end{array}$ & $\begin{array}{c}\text { Absorbancia } \\
\text { ELISA }\end{array}$ \\
\hline $10^{6}$ prom IV & 1 & $2 \mathrm{mpi}$ & + & - & $1: 64$ & 0.268 \\
\hline $10^{6}$ prom IV & 1 & $4 \mathrm{mpi}$ & + & $\mathrm{nLp}$ & $1: 256$ & 1.266 \\
\hline $10^{6}$ prom IV & 1 & $8 \mathrm{mpi}$ & $\sim$ & $\sim$ & $\sim$ & $\sim$ \\
\hline $10^{6}$ prom IV & 2 & $2 \mathrm{mpi}$ & - & - & $1: 16$ & 0.222 \\
\hline $10^{6}$ prom IV & 2 & $4 \mathrm{mpi}$ & - & - & $1: 128$ & 0.326 \\
\hline $10^{6}$ prom IV & 2 & $8 \mathrm{mpi}$ & - & - & $1: 16$ & 0.279 \\
\hline $10^{6}$ prom IV & 3 & $2 \mathrm{mpi}$ & - & - & $1: 128$ & 0.994 \\
\hline $10^{6}$ prom IV & 3 & $4 \mathrm{mpi}$ & + & $\mathrm{nLp}$ & $1: 256$ & 1.500 \\
\hline $10^{6}$ prom IV & 3 & $8 \mathrm{mpi}$ & $\sim$ & $\sim$ & $\sim$ & $\sim$ \\
\hline $10^{4}$ prom IV & 4 & $2 \mathrm{mpi}$ & - & - & NR & 0.338 \\
\hline $10^{4}$ prom IV & 4 & $4 \mathrm{mpi}$ & + & $\mathrm{nLp}$ & NR & $>2$ \\
\hline $10^{4}$ prom IV & 4 & $8 \mathrm{mpi}$ & + & - & $1: 256$ & 1.65 \\
\hline $10^{4}$ prom IV & 5 & $2 \mathrm{mpi}$ & - & - & $1: 16$ & 0.208 \\
\hline $10^{4}$ prom IV & 5 & $4 \mathrm{mpi}$ & - & - & $1: 64$ & 0.419 \\
\hline $10^{4}$ prom IV & 5 & $8 \mathrm{mpi}$ & - & - & NR & 0.432 \\
\hline $10^{4}$ prom IV & 6 & $2 \mathrm{mpi}$ & - & - & $1: 128$ & 0.362 \\
\hline $10^{4}$ prom IV & 6 & $4 \mathrm{mpi}$ & - & $\mathrm{nLp}$ & $1: 256$ & 0.738 \\
\hline $10^{4}$ prom IV & 6 & $8 \mathrm{mpi}$ & - & - & $1: 128$ & 0.921 \\
\hline $10^{4}$ prom IV & 7 & $2 \mathrm{mpi}$ & - & - & $1: 16$ & 0.384 \\
\hline $10^{4}$ prom IV & 7 & $4 \mathrm{mpi}$ & - & $\mathrm{nLp}$ & $1: 16$ & 0.189 \\
\hline $10^{4}$ prom IV & 7 & $8 \mathrm{mpi}$ & - & $\mathrm{nLp}$ & $1: 64$ & 0.262 \\
\hline $10^{4}$ prom ID & 8 & $2 \mathrm{mpi}$ & - & - & $1: 32$ & 0.311 \\
\hline $10^{4}$ prom ID & 8 & $4 \mathrm{mpi}$ & + & $\mathrm{nLp}$ & $1: 128$ & 0.719 \\
\hline $10^{4}$ prom ID & 8 & $8 \mathrm{mpi}$ & + & $\mathrm{nLp}$ & $1: 256$ & 1.512 \\
\hline $10^{4}$ prom ID & 9 & $2 \mathrm{mpi}$ & + & - & $1: 32$ & 0.253 \\
\hline $10^{4}$ prom ID & 9 & $4 \mathrm{mpi}$ & + & - & $1: 128$ & 0.612 \\
\hline $10^{4}$ prom ID & 9 & $8 \mathrm{mpi}$ & + & - & $1: 32$ & 1.216 \\
\hline $10^{4}$ prom ID & 10 & $2 \mathrm{mpi}$ & + & - & $1: 16$ & 0.362 \\
\hline $10^{4}$ prom ID & 10 & $4 \mathrm{mpi}$ & + & - & $1: 64$ & 0.267 \\
\hline $10^{4}$ prom ID & 10 & $8 \mathrm{mpi}$ & + & $\mathrm{nLp}$ & $1: 64$ & 0.267 \\
\hline Inf. natural & 11 & $\sim$ & + & $\mathrm{nLp}$ & $1: 128$ & 1.5 \\
\hline Inf. natural & 12 & $\sim$ & + & $\mathrm{nLp}$ & $1: 4096$ & 2.5 \\
\hline Inf. natural & 13 & $\sim$ & + & $n L p$ & $1: 4096$ & 2.443 \\
\hline Inf. natural & 14 & $\sim$ & + & $\mathrm{nLp}, \mathrm{B}$ & $1: 4096$ & 1.434 \\
\hline Inf. natural & 15 & $\sim$ & + & B & $1: 512$ & 1.5 \\
\hline
\end{tabular}

IV: Invtravenoso. ID: Intradérmico. nLp: Nódulo linfático poplíteo. B: Bazo

Por WB, los sueros de los perros infectados experimentalmente con $L$. infantum reconocieron 15 fracciones antigénicas entre 10 y $123 \mathrm{kDa}$ mientras que los perros infectados naturalmente reconocieron 9 fracciones antigénicas adicionales. Los perros infectados con T. cruzi mostraron reacciones cruzadas en 8 bandas. Los animales infectados con Erhlichia spp., Babesia spp. y Dirofilaria spp. reconocieron únicamente las bandas de 50 y $69 \mathrm{kDa}$; en el caso de los perros infectados con L. (Viannia) spp. no se observó reacción cruzada con la fracción antigénica de $13 \mathrm{kDa}$, la cual fue reconocida exclusivamente en sueros de perros infectados con L. infantum. Los perros sanos reconocieron las bandas de 69, 75, y $123 \mathrm{kDa}$. 
Se encontró que el $64 \%$ de los perros sintomáticos y el $37 \%$ de los asintomáticos fueron positivos a WB. En muestras de perros parasitológicamente confirmados el $77 \%$ fueron positivas a WB mientras que el $45 \%$ fueron positivas para WB, pero negativas parasitológicamente a pesar de la infección experimental.

En los animales infectados experimentalmente con L. infantum se encontró que el $45,4 \%$ fueron positivos a IFI y WB y que el $67,5 \%$ de los perros no infectados fueron negativos a las dos pruebas. Por ELISA y WB se encontró que para estas dos pruebas de los animales infectados experimentalmente fueron positivos el 48,4 \% y negativos el 57,5\% de los perros no infectados.

Tabla 2. Parámetros calculados para la evaluación del estado clínico, parasitológico y las pruebas serológicas de IFI, ELISA y WB en los casos de leishmaniasis visceral canina

\begin{tabular}{|c|c|c|c|c|c|}
\hline \multirow[b]{2}{*}{$\begin{array}{l}\text { Parametro } \\
\text { Evaluado }\end{array}$} & \multicolumn{5}{|c|}{ Pruebas Diagnósticas Empleadas } \\
\hline & $\begin{array}{c}\text { Estado } \\
\text { Clinico } \\
\% \\
(\mathrm{IC})^{*}\end{array}$ & $\begin{array}{c}\text { Examen } \\
\text { Parasitologico } \\
\% \\
(\mathrm{IC})^{\star}\end{array}$ & $\begin{array}{c}\text { IFI } \\
\% \\
(I C)^{\star}\end{array}$ & $\begin{array}{c}\text { ELISA } \\
\% \\
(I C)^{\star}\end{array}$ & $\begin{array}{l}\text { WB } \\
\% \\
(I C)^{*}\end{array}$ \\
\hline Sensibilidad & $\begin{array}{c}51.5 \\
(34.5-68.6)\end{array}$ & $\begin{array}{c}39.4 \\
(22.7-56.1)\end{array}$ & $\begin{array}{r}69.7 \\
(54.1- \\
85.4)\end{array}$ & $\begin{array}{c}51.5 \\
(34.5-68.6)\end{array}$ & $\begin{array}{c}57.6 \\
(40.7-74.4)\end{array}$ \\
\hline Especificidad & $\begin{array}{c}50 \\
(34.5-65.5)\end{array}$ & $\begin{array}{c}100 \\
-\end{array}$ & $\begin{array}{r}67.5 \\
(52.9- \\
82.1)\end{array}$ & $\begin{array}{c}57.5 \\
(42.2-72.8)\end{array}$ & $\begin{array}{c}100 \\
-\end{array}$ \\
\hline $\begin{array}{c}\text { Valor Predictivo } \\
\text { Positivo }\end{array}$ & $\begin{array}{c}45.9 \\
(29.9-62)\end{array}$ & $\begin{array}{c}100 \\
-\end{array}$ & $\begin{array}{c}63.8 \\
(48.2- \\
79.6)\end{array}$ & $\begin{array}{c}50 \\
(33.1-66.8)\end{array}$ & $\begin{array}{c}100 \\
-\end{array}$ \\
\hline $\begin{array}{c}\text { Valor Predictivo } \\
\text { Negativo }\end{array}$ & $\begin{array}{c}55.6 \\
(39.3-71.8)\end{array}$ & $\begin{array}{c}66.7 \\
(34.5-65.5)\end{array}$ & $\begin{array}{r}72.9 \\
(58.6- \\
87.3)\end{array}$ & $\begin{array}{c}58.9 \\
(43.5-74.4)\end{array}$ & $\begin{array}{c}74.1 \\
(62.4-85.8)\end{array}$ \\
\hline $\begin{array}{l}\text { Prevalencia } \\
\text { Aparente }\end{array}$ & $\begin{array}{c}50.7 \\
(39.2-62.2)\end{array}$ & $\begin{array}{c}17.8 \\
(9.03-26.6)\end{array}$ & $\begin{array}{c}49.3 \\
(37.8- \\
60.8)\end{array}$ & $\begin{array}{c}46.6 \\
(35.1-58.1)\end{array}$ & $\begin{array}{c}26.1 \\
(16.1-36.1)\end{array}$ \\
\hline Prevalencia Real & & 45 & $33.8-56$. & & \\
\hline
\end{tabular}

Los resultados y los parámetros de todas las pruebas pueden observarse en la Tabla 2. El examen parasitológico mostró la menor sensibilidad y las prevalencias aparentes obtenidas mediante las pruebas de WB y el examen parasitológico son las más bajas, además la prevalencia obtenida mediante el examen parasitológico difiere significativamente $(\alpha=0,05)$ de la prevalencia real. Los índices kappa $(\kappa)$ más altos se presentaron con la prueba de IFI y ELISA $(\kappa=0,59)$, examen parasitológico y estado clínico $(\kappa=0,59)$ y examen parasitológico y WB $(\kappa=0,52)$. 


\section{DISCUSIÓN}

El desarrollo de herramientas diagnósticas para detectar animales infectados con L. infantum constituye una de las tareas prioritarias establecidas por la OMS para el control de la leishmaniasis zoonótica (1,32). De Paula et al. (3) e Iniesta et al. (17) afirman que los estudios epidemiológicos y las medidas de control, en los casos de LV zoonótica, deben basarse en los valores verificados de sensibilidad y especificidad de cada prueba, sin embargo, no existe consenso sobre cual técnica sería la más eficiente.

Según Desjeux (1), en humanos el diagnóstico por aspirado de bazo es considerado el "gold estándar". Esto contrasta con lo obtenido en los perros infectados naturalmente donde se encontró que la impronta de bazo fue menos sensible (40\%) que el aspirado de ganglio linfático poplíteo (80 \%). En los perros experimentales la máxima sensibilidad del aspirado de ganglio alcanzó el $70 \%$ a los 4 mpi. Es posible que existan variaciones con respecto a la distribución del parásito en relación a la especie afectada (4,33-35).

Alexandre y Dias (9) reportan que IFI mejora la precocidad del diagnóstico en perros asintomáticos situación que se confirmó experimentalmente en perros infectados pero negativos parasitológicamente. De los 10 sueros tomados 2 mpi el $50 \%$ fue positivo por IFI, $20 \%$ por WB y solo el $10 \%$ por ELISA. Los programas de control de LV canina han considerado la prueba de IFI como la técnica de elección para el diagnóstico $(9,10,32,36)$; en este estudio se encontró que el 27,3\% de los perros infectados mostraron títulos negativos (Tabla 1). Bernardina et al. (13) y Mancianti et al. (6) reportaron positividad para la prueba de IFI entre el $93 \%$ y el 97,8\% en perros confirmados parasitológicamente respectivamente, datos que contrastan con el $72,7 \%$ de positividad obtenido en este estudio. Por IFI solo se detectó al 67,5\% de los perros no infectados como tal, similar a lo descrito por De Paula et al. (3).

En los perros se han reportado reacciones cruzadas por IFI con miembros relacionados de la familia Trypanosomatidae como T. cruzi y algunas especies de Leishmania sp. cutánea circulantes en perros $(9,32)$. Vega et al. (10) encontraron reacciones cruzadas con $T$. evansi y el presente trabajo evidenció positividad después de los 2 mpi en perros con T. cruzi. Por IFI no se encontraron reacciones cruzadas con babesiosis, erhlichiosis ni dirofilariasis, similar a lo descrito por Mancianti et al. (6). 
Según Alexandre\&Dias (9), Vega et al. (10), Travi et al. (4) y Mancianti et al. (37) la prueba de ELISA ha permitido obtener mayor sensibilidad y especificidad en el diagnostico de LV canina. Sin embargo, el ELISA no mejoró la precocidad en el diagnóstico con respecto a IFI. Según Nieto et al. (22) el tipo de antígeno y la purificación del mismo puede afectar los resultados en fases iniciales de la infección con LV canina. El porcentaje de positividad obtenido en los perros infectados tanto natural como experimentalmente, fue del 51,5\%, el menor para las tres pruebas utilizadas, lo que contrasta con lo descrito por Mancianti et al. (37). Por ELISA se obtuvo el mayor porcentaje de falsos positivos y de los perros no infectados el $10 \%$ no mostraron títulos de anticuerpos por IFI pero sí fueron positivos por ELISA; lo que concuerda con lo descrito por Almeida et al. (7) quienes encontraron que los valores de especificidad de ELISA disminuyeron debido al uso de antígenos crudos y la presencia de anticuerpos de reacción cruzada. En los sueros de perros inoculados con T. cruzi el $81 \%$ fueron positivos por ELISA, reacciones que también han sido reportadas Vercammen et al. (12). Se observó reacción cruzada con Erhlichia spp. a diferencia de lo reportado por Mancianti et al. (6) quienes encontraron reacciones cruzadas con Dirofilaria spp. y Babesia spp. Las reacciones inespecíficas pueden darse con antígenos crudos como lo reportan Alexandre\&Dias (9) y Mancianti et al. (37) o a condiciones nutricionales y exposición a otros patógenos como lo reportan Bernardina et al. (13), Abranches et al. (18) y Pappas et al. (26).

La prueba de WB no incrementó significativamente los niveles de detección obtenidos por las pruebas de IFI o ELISA en los animales infectados. Los resultados de las pruebas de ELISA y WB en animales infectados fueron similares, datos que concuerdan con los reportados por Deplazes et al. (38), posiblemente debido a que el antígeno empleado para las dos pruebas fue el mismo y el porcentaje de detección de perros en fases iniciales de la infección experimental fue bajo para las dos pruebas. Teniendo en cuenta que con la prueba de WB fue posible determinar las fracciones antigénicas responsables de algunas de las reacciones cruzadas que probablemente afectaron el desempeño de las pruebas de IFI y ELISA, se logró disminuir el número de falsos positivos por WB.

Se encontró que las fracciones de 13, 57 y 73 kDa se visualizaron en los sueros de perros parasitológicamente comprobados e infectados naturalmente y en los sueros de seguimiento experimental en todas las fases y con todos los inóculos. Datos similares han sido reportados por De Paula et al. (3), Aisa et al. (16), Mancianti et al. (37), Neogy et al. (15) y Abranches et al. (18). 
Fracciones de bajo peso molecular $(13 \mathrm{kDa})$ han sido reportadas por Mary et al. (39) como potencialmente diagnósticas, especialmente en fases tempranas de LV, dato que corresponde a lo observado en este estudio donde esta banda fue reconocida solamente por los perros inoculados experimentalmente con $L$. infantum a partir de los 2 mpi e incluso no fue reconocida por los sueros de perros infectados con L. (Viannia) spp. Fernández-Pérez et al. (8) reportaron la visualización de la banda de $57 \mathrm{kDa}$ en perros sintomáticos relacionándola con respuesta tipo Th2, por el contrario esta banda fue reconocida en el presente estudio tanto en perros sintomáticos como asintomáticos, posiblemente porque durante el proceso de infección los animales se encontraban en fase prepatente. Con respecto al reconocimiento de la banda de $73 \mathrm{kDa}$, Mancianti et al. (37) reporta la presencia de esta banda en perros infectados, sin embargo Mary et al. (32) y Aisa et al. (16) describen reacciones inespecíficas con los antígenos ubicados en el rango de 70-75 $\mathrm{kDa}$ en perros de zonas libres de LV. La alta frecuencia de reconocimiento y la fuerte intensidad que se presentó con la banda de $69 \mathrm{kDa}$ pudo deberse a que, como lo expone Aisa et al. (16), en este rango de pesos moleculares se encuentran péptidos de proteínas de choque térmico, altamente conservados entre los diferentes organismos vivos. En este trabajo se encontraron reacciones cruzadas con antígenos de Babesia spp., específicamente con las fracciones de 50, 69 y $75 \mathrm{kDa}$, sin embargo las reacciones cruzadas con antígenos de Ehrlichia spp. descritas en este mismo trabajo con la prueba de ELISA no fueron confirmadas por prueba de WB.

Con respecto a otras bandas importantes encontradas, la banda de $32 \mathrm{kDa}$ ha sido también descrita por De Paula et al. (3) quienes la catalogan como potencialmente diagnóstica en los casos de LV en América asociándola con una banda de $29 \mathrm{kDa}$, que también fue visualizada en los perros infectados con $L$. infantum en todos los inóculos y durante todo el seguimiento experimental pero de forma similar fue observada en una muestra de suero de uno de los perros inoculados experimentalmente con T. cruzi 120 dpi. Por lo tanto, los hallazgos obtenidos indican que en zonas donde circulen de forma concomitante $L$. infantum y T. cruzi no debería tenerse en cuenta las bandas de 29, 34, 50, 69, 75, 86, 99 y $123 \mathrm{kDa}$. El uso de los análisis de WB es útil en la determinación de los perros infectados con LV; sin embargo no existe consenso con respecto al patrón de reconocimiento.

Los estudios realizados en Brasil, muestran a IFI como la prueba de referencia para el control de LV en perros $(3,9,40)$. Los resultados de este trabajo confirmarían la utilidad de la prueba de IFI ya que mostró la mayor sensibilidad y una alta especificidad comparada con las demás pruebas, adicionalmente junto 
con la prueba de ELISA fue la que más se acercó a la prevalencia real. La concordancia $(\kappa)$ más alta a nivel de campo se presentó con las pruebas de IFI y WB, lo que indicaría que al emplearlas concomitantemente sería posible obtener una buena sensibilidad (IFI) y especificidad (WB) en los análisis de campo como lo demostró De Paula et al. (3) y las prevalencias aparentes obtenidas no se alejarían significativamente de la prevalencia real.

Teniendo en cuenta que los métodos directos y las pruebas serológicas en zonas endémicas pueden subdiagnosticar la infección por LV es recomendable adaptar nuevas técnicas que permitan un diagnóstico más sensible en las etapas tempranas de la infección y después del tratamiento en los perros afectados; sin embargo, solamente un constructo que combine tanto pruebas directas como indirectas sería la forma más eficiente de diagnóstico. En el caso de WB, el emplear las bandas específicas encontradas en este trabajo como indicadores de infección temprana, especialmente aquellas con pesos moleculares $<30 \mathrm{kDa}$, con preparaciones antigénicas mucho más elaboradas, podría mejorar los valores de sensibilidad y especificidad de la prueba. Debido a que existen otras formas zoonóticas de leishmaniasis circulando en los perros en América, en estudios posteriores deberían emplearse un mayor número de muestras de suero de perros infectados con aislados causantes de leishmaniasis cutánea, ya que los datos obtenidos en este estudio aún son muy reducidos

\section{REFERENCIAS}

1. Desjeux P. Leishmaniasis: current situation and new perspectives. Comp Immunol Microbiol Infect Dis 2004; 27:305-318.

2. Tesh R. Control of zoonotic visceral leishmaniasis: Is it time to change strategies? Am J Trop Med Hyg 1995; 52: 287-292.

3. De Paula A, Da Silva A, Fernandes O, Jansen A. The use of immunoblot analysis in the diagnosis of canine visceral leishmaniasis in an endemic area of Rio de Janeiro. J Parasitol 2003; 89: 832-836.

4. Ciaramella P \& Corona M. Canine leishmaniasis: Clinical and diagnostic aspects. Comp Cont Educ Pract Vet 2003; 25:358-369.

5. Travi B, Tabares C, Cadena H, Ferro C, Osorio Y. Canine visceral leishmaniasis in Colombia. Relationship between clinical and parasitological status and infectivity for sand flies. Am J Trop Med Hyg 2001; 64:119-124.

6. Mancianti F, Pedonese F, Poli A. Evaluation of dot-enzyme linked immunoabsorbent assay (dotELISA)for the serodiagnsosis of canine leishmaniasis as compared with indirect immunofluorescence assay. Vet Parasitol 1996; 65:1-9.

7. Almeida M, Jesus E, Sousa-Atta M, Alves L, Berne M, Atta A. Antileishmanial antibody profile in dogs naturally infected with Leishmania chagasi. Vet Immunol Immunopatol 2005; 106:151158.

8. Fernandez-Perez F, Gómez-Muñoz S, Mendez J. Leishmania-specific lymphoproliferative responses and lg G1/lg G2 immunodetection patterns by Western blot in asymptomatic, symptomatic and treated dogs. Acta Trop. 2003; 86:83-91. 
9. Alexandre W \& Dias P. Reflexões sobre a qualidade do diagnóstico da leishmaniose visceral canina em inquéritos epidemiológicos: o caso da epidemia de Belo Horizonte, Minas Gerais, Brasil, 1993-1997. Cad Saúde Pública, Rio de Janeiro 2004; 20:259-265.

10. Vega J, López C, Vargas J, Ayala M. Nicholls S, Bello F, et al. Estandarización de la prueba de ELISA para el inmunodiagnóstico de la Leishmaniasis visceral canina. In: Agudelo C. eds. I Encuentro de Investigadores en Salud Pública de la Universidad Nacional de Colombia. Bogotá: Instituto de Salud Pública; 2003.

11. Riera C, Valladares J, Gallego M, Aisa M, Castillejo S, Fisa R, et al. Serological and parasitological follow-up in dogs experimentally inected with Leishmania infantum and treated with meglumine antimoniate. Vet Parasitol 1999; 84:33-47.

12. Vercammen F, Berkvens D, Le Ray D, Jacquet D, Vervoort T. Development of a slide ELISA for canine leishmaniasis and comparison with four serological tests. Vet Record 1997; 141: 328-330.

13. Bernadina W, De Luna R, Oliva G, Ciaramella P. An immunodiffusion assay for the detection of canine leishmaniasis due to infection with Leishmania infantum. Vet Parasitol 1997; 73:207-213.

14. Harith A, Slappendel R, Reiter I, Van Knapen F, De Korte P, Huigen E, Kolk A. Application of a direct agglutination test for the detection of specific anti- Leishmania antibodies in the canine reservoir. J Clin Microbiol 1989; 27:2252-2257.

15. Neogy A, Vouldoukis I, Silva O, Tselentis Y, Lascombe J, Segalen T, et al. Serodiagnosis and screening of canine visceral leishmaniasis in an endemic area of Corsica: Aplicability of a direct agglutination test and immunoblot analysis. Am J Trop Med Hyg 1992; 47:772-777.

16. Aisa M, Castillejo S, Gallego M, Fisa R, Riera M, Colmenares M et al. Diagnostic potential of western blot analisis of sera from dogs with Leishmaniasis in endemic areas and significance of the pattern. Am J Trop Med Hyg 1998; 58:154-159.

17. Iniesta L, Fernandez-Barredo S, Bulle B, Gomez M, Piarrous R, Gallego M, et al. Diagnostic techniques to detect cryptic leishmaniasis in dogs. Clin Diagn Lab Immunol 2002; 9:11371141.

18. Abranches P, Santos-Gómes G, Rachamim N, Campino L, Schnur L, Jaffe C. An experimental model for canine visceral leishmaniasis. Parasite Immunol 1991; 13:537-550.

19.Rolland L, Monjour M, Danis M, Gentilini M. Leishmanioses viscérale. Diagnostic par immunoempreinte. Presse Médicale $1992 ; 21: 971-973$.

20. Carrera L, Fermin L, Tesouro M, García P, Rolla E, Gonzalez J, et al. Antibody response in dogs experimentally infected with Leishmania infantum: Infection course antigen markers. Experimental Parasitol 1996; 82:139-146.

21. Correira Da Costa J, Asit B, Vouldoukins I., Sampaio M, Gentilini M, Monjour L. Antigenic components of partially purified antigens of Leishmania donovani infantum reocognized by sera from dogs with asymptomatic or active visceral leishmaniasis. Am J Trop Med Hyg 1996; 55:511-515.

22. Nieto CG, Garcia-Alonso M, Requena J, Miron C, Soto M, Alonso C, Navarrete I. Analysis of the humoral immune response against total and recombinant antigens of Leishmania infantum correlation with disease progression in canine experimental leishmaniasis. Vet Immunol Immunopathol 1999; 67:117-130.

23. Lasri S, Sahibi H, Natami A, Rhalem A. Western blot analysis of Leishmania infantum antigens using sera from pentamidine-treated dogs. Vet Immunol Immunopathol 2003; 91:13-18.

24. Iniesta L, Gallego M, Portus M. Immunoglobulin $G$ and responses in varios stages of canine leshmaniosis. Vet limmunol limmunopathol 2005; 103:77-81.

25. Corredor A, Alvarez C, Agudelo C, López C, Cáceres M, Reyes P, et al. Prevalence of Trypanosoma cruzi and Leishmania chagasi infections risk factors in Colombian indigenous population. Rev Inst Med Trop S Paulo 1999; 41:229-234.

26. Pappas M, Hajkowski R, Hockmeyer T. Standardization of the Dot-Enzyme-Linked immunosorbent Assay (Dot-ELISA) for human visceral leishmaniasis. Am J Trop Med Hyg 1984; 33:11051111. 
27. Laemmli U. Cleavage of structural proteins during the assembly of the head of bacteriophage T4. Nature 1970; 227:680-685.

28. Towbin H, Staehelin T, Gordon J. Electrophoretic transfer of proteins from polyacrilamide gels to nitrocellulose sheets: procedures and some applications. Proc Natl Acad Sci U S A 1979; 76:4350-4354.

29. Bradford M. A rapid and sensitive method for the quantification of microgram quantities of protein utilizing the principle of protein dye binding. Anal Biochem 1976; 72:248-254.

30. Congreso de la República de Colombia. Ley 084 de 1989. Estatuto Nacional de Protección de los animales. Diario Oficial, Año CXXVI. N.39120.27,1989:1-14.

31. Ministerio de Salud, República de Colombia. Resolución No. 008430 de 1993. Investigación en Salud. 1993:1-20.

32. Cabrera M, Paula A, Camacho L, Marzochi M, Navier S, Da Silva A, et al. Canine visceral leishmaniasis in Barra de Guarativa, Rio de Janerio, Brazil: Assessment of risk factors. Rev Inst Med Trop S Paulo 2003; 45:79-83.

33. Ashford R. The leishmaniasis as model zoonoses. Ann Trop Med Parasitol 1997; 91:693-701.

34. Miles M, Vexanat J, Furtado Campos H, Fonseca de Castro J. Canine leishmaniasis in Latin America: Control strategies for visceral leishmaniasis. In: Hoechst Roussel Vet. Canine Leishmaniasis: An update. Proceedings of the Internacional Canine Leishmaniasis forum. Barcelona Hoechst Roussel Vet; 1999.

35. Molina R, San Andres M, Tesouro M, Nieto J, Vitututia M, Gonzalez F, et al. Canine leishmaniaisis: clinical, parasitological and entomological follow up after chemotherapy. Ann Trop Med Parasitol 1994; 88: 371-378.

36. Fernandez J, Charry T, Lozano C, Rojas C, Mazabel C, Bello F, et al. Prevalencia de Leishmaniasis Visceral Canina en Municipios de Huila-Colombia. Rev Salud Pública 2003; 4:278-285.

37. Mancianti F, Falcone M, Giannelli C, Poli A. Comparison between an enzyme linked immunosorbent assay using a detergent soluble Leishmania infantum antigen and indirect immunofluorescence for the diagnosis of canine leishmaniasis. Vet Parasitol 1995; 59:13-21.

38. Deplazes P, Smith N, Arnold P, Lutz H, Eckert J. Specific Ig G1 and Ig G2 antibody responses of dogs to Leishmania infantum and others parasites. Parasite immunol 1995; 17: 451-458.

39. Mary C, Lamouroux D, Dunan S, Quilici M. Western blot analysis of antibodies to Leishmania infantum antigens: potential of the $14-\mathrm{Kd}$ and $16-\mathrm{Kd}$ antigens for diagnosis and epidemiological purposes. Am J Trop Med Hyg 1992; 47:764-771.

40. De Oliveira S, Araujo M. Avaliacao das acoes de controle da leishmaniose visceral (calazar) em uma area endemica do Estado da Bahia, Brasil (1995-2000). Cad. Saúde Pública, Rio de Janeiro 2003; 51:1681-1690. 